\title{
Magnetic control of stem cells: feasible for ED
}

...MN-ADSCs were retained within the corpus cavernosa for up to 3 days...
Intracavernosal injection of stem cells has been suggested as a treatment of erectile dysfunction (ED); however, rapid washout of stem cells following injection has, thus far, hindered progress of research in this area. Now, findings of a preclinical study in rats demonstrate that magnetic, nanoparticle-bound, adipose-derived stem cells (MN-ADSCs) - which enable magnetic control of stem-cell location - can be succesfully retained within the corpora cavernosa.

Researchers tested the effectiveness of MN-ADSC injections in a rat bilateral cavernous nerve crush (BCNC) model, which was intended to mimic the effects of radical prostatectomy. Following
MN-ADSC injection, with magnetic force applied outside of the corpus cavernosum for a duration of $6 \mathrm{~h}$, MN-ADSCs were retained within the corpus cavernosum for up to 3 days, confirmed by the presence of a fluorescent signal, with which all ADSCs were labelled before injection. However, rats that received either non-nanoparticle bound ADSCs, or MN-ADSCs without subsequent use of the magnet had few detectable ADSCs 1 day after injection.

The ratio of intracavernosal to mean arterial pressure, measured under stimulation of the cavernous nerve, was significantly higher in those rats injected with MN-ADSCs that were also exposed to localized magnetic force, suggesting an improvement in erectile function in this group relative to either control group.

These findings suggest that ADSCs injected into the corpus cavernosum can be bound to magnetic nanoparticles and retained by subsequent use of a magnet. Longer-term in vivo data on safety, tolerability and functional outcomes are eagerly awaited.

Peter Sidaway

ORIGINAL ARTICLE Lin, H. et al. Nanoparticle improved stem cell therapy for erectile dysfunction in a rat model of cavernous nerve injury. J. Urol. http://dx.doi.org/10.1016/ j.juro.2015.10.129 